\title{
Application of the Regularization Strategy in Solving Numerical Differentiation for Function with Error
}

\author{
Xian-Zhou GUO ${ }^{1, a}$ and Xiang-Mei ZHANG ${ }^{2, b, *}$ \\ ${ }^{1}$ Hebei University of Technology, Tianjin City, 300401, P.R. China \\ ${ }^{2}$ Hebei University of Technology, Tianjin City, 300401, P.R. China \\ axianzhou_guo@hebut.edu.cn, bxmei_zhang@hebut.edu.cn \\ ${ }^{*}$ Corresponding author
}

Keywords: Noisy function, Exact function, Differentiation, Regularization strategy, Regularization parameter.

\begin{abstract}
In many practical problems, it is sometimes necessary to evaluate the derivative of function whose values are given approximately. Firstly, the problem of estimating the derivative of a function observed with error is studied. It presents a proper regularization strategy and explain how to choose regularization parameter. Secondly, the regularization strategy above to the numerical differentiation is applied and discussed in the implementation of the numerical method and the tests which it has performed in order to investigate the accuracy and stability of the numerical differentiation procedure. Finally, some numerical examples will further illustrate that this method is reasonable, effective and reasonable.
\end{abstract}

\section{Introduction}

In recent years, inverse problems in mathematical physics have been one of the fastest growing areas. However, inverse problems are closely linked to the ill-posed, and due to a great deal of difficulty to numerical solution. At the beginning of 1960s, the regularization strategy is proposed by Tikhonov creatively[1]. From then on, Dinh Nho Hao studied the mollification method for ill-posed problems[2]. Y F Wang, W Q Liang, J T Zhao conducted a lot of research[3,4,5]. As it is known, the choice of the regularization parameter is a key matter for ensuring proper regularization. There are many problems that can be described by numerical differentiation in the natural science and engineering technology field. It is easy to imagine many different situations--- mostly involving ordinary and partial differential equations - related with the question of numerical differentiation of noisy (measured) data. So it is sometimes necessary to evaluate the derivative of function whose values are given approximately. How to get the numerical solution of these functions with noisy data has become a special course. In this paper, the operator with Gaussian kernel is studied and its reasonable regularization parameters in an efficient manner is introduced.

Therefore, a lot of numerical examples show that the process has good stability and high accuracy. The general method to solve the ill-posed problem is to approximate the solution of the original problem with a set of well-posed problems. How to establish an effective regularization method is an important part of the research on the problem of ill-posed problems in the field of inverse problem. $\mathrm{J}$ J Cao, Y F Wang and B F Wang[6,7] conducted a lot of research.

\section{Regularization Strategy}

Theorem 1: Let $g_{\alpha} \in L^{1}\left(R^{n}\right)$ and $\int_{R^{n}} g_{\alpha}(x) d x=1$,

(1) if $f \in L^{p}\left(R^{n}\right)$, where $1 \leq p<+\infty$, then $\lim _{\alpha \rightarrow 0}\left\|g_{\alpha} * f-f\right\|_{p}=0$;

(2) if $f \in L^{\infty}\left(R^{n}\right)$, then $\lim _{\alpha \rightarrow 0}\left(g_{\alpha} * f\right)(x)=f(x)$, where $\mathrm{x}$ is the continuous point of $f$.

Proof: Set $g_{\alpha} * f=f_{\alpha}, f_{\alpha}(x)-f(x)=\int_{R^{n}}[f(x-y)-f(x)] g_{\alpha}(y) d y$. 
By the generalized Minkowski inequality,

$$
\left\|f_{\alpha}-f\right\|_{p}=\left\{\int_{R^{n}}\left|\int_{R^{n}}[f(x-y)-f(x)] g_{\alpha}(y) d y\right|^{p} d x\right\}^{1 / p} \leq \int_{R^{n}}\left[\iint_{R^{n}}|f(x-\alpha t)-f(x)|^{p} d x\right]^{1 / p}|g(t)| d t .
$$

To every $t \in R^{n}$,

$$
\lim _{\alpha \rightarrow 0}\left[\int|f(x-\alpha t)-f(x)|_{R^{n}}^{p} d x\right]^{1 / p}=0
$$

and

$$
\left[\int_{R^{n}}|f(x-\alpha t)-f(x)|^{p} d x\right]^{1 / p} \leq 2\|f\|_{p}
$$

By dominated convergence theorem,

$$
\int_{R^{n}}\left[\int_{R^{n}}|f(x-\alpha t)-f(x)|^{p} d x\right]^{1 / p}|g(t)| d t \rightarrow 0 .
$$

When $\alpha \rightarrow 0$. (1) has already proved.

Because $f \in L\left(R^{n}\right)$ and $x$ is a continuous point of $f$, then

$$
\left|f_{\alpha}(x)-f(x)\right| \leq \int_{R^{n}}\left|(f(x-y)-f(x)) g_{\alpha}(y)\right| d y=\int_{R^{n}}|f(x-\alpha t)-f(x)||g(t)| d t .
$$

To every $t \in R^{n}$,

$$
\lim _{\alpha \rightarrow 0}|f(x-\alpha t)-f(x)|=0
$$

and

$$
|f(x-\alpha t)-f(x)| \leq 2\|f\| .
$$

By dominated convergence theorem,

$$
\int_{R^{n}}|f(x-\alpha t)-f(x)||g(t)| d t \rightarrow 0 \text {, when } \alpha \rightarrow 0 .
$$

(2) has already proved.

By Theorem 1, $g_{\alpha}$ is needed to choose properly, then $g_{\alpha} * f \rightarrow f$ when $\alpha \rightarrow 0$.

The Gaussian kernel $g_{\alpha}(t)$ is defined by $g_{\alpha}(t)=\frac{1}{\alpha \sqrt{\pi}} \exp \left(-t^{2} / \alpha^{2}\right), t \in R$, where $\alpha>0$ denotes a parameter. Then $\int_{-\infty}^{+\infty} g_{\alpha}(t) d t=1$ and the convolution 
$T_{\alpha} f(t)=\left(g_{\alpha} * f\right)(t)=\int_{-\infty}^{+\infty} g_{\alpha}(t-s) f(s) d s=\int_{-\infty}^{+\infty} g_{\alpha}(s) f(t-s) d s, t \in R$

exists and is an $L^{2}$-function for every $f(t) \in L^{2}(R)$. Furthermore, by Young’s inequality,

$\left\|T_{\alpha} f\right\|_{L^{2}}=\left\|g_{\alpha} * f\right\|_{L^{2}} \leq\left\|g_{\alpha}\right\|_{L^{1}}\|f\|_{L^{2}}=\|f\|_{L^{2}}, \forall f \in L^{2}(R)$ is obtained.

Therefore, the operator $f \rightarrow g_{\alpha} * f$ is uniformly bounded in $L^{2}(R)$ with respect to $\alpha$. So regard $T_{\alpha} f(t)$ as regularization operator.

However, in practical application, $f(t)$ cannot be accurately given. The noisy (measured) function $f_{\delta}(t)$ which satisfied the error bound is obtained:

$$
\left\|f(t)-f_{\delta}(t)\right\|_{\infty, I} \leq \delta
$$

in the data interval $I=[0,1]$.

Theorem 2: ( Error estimation):

$$
\begin{aligned}
\left|T_{\alpha} f_{\delta}(t)-f(t)\right| & =\left|T_{\alpha} f_{\delta}(t)-T_{\alpha} f(t)+T_{\alpha} f(t)-f(t)\right| \\
& \leq\left|T_{\alpha} f_{\delta}(t)-T_{\alpha} f(t)\right|+\left|T_{\alpha} f(t)-f(t)\right| \leq \delta+C \alpha
\end{aligned}
$$

where $\alpha$ is a constant. So $T_{\alpha} f_{\delta}(t)$ is used to compute the approximate derivative of the exact $f(t)$.

The original ill-posed problem of finding $f$ is replaced by new problem of finding $T_{\alpha} f_{\delta}$. The new problem is well-posed, and depends on a parameter $\delta>0$.

For given $T_{\alpha} f_{\delta}$, regularization parameter $\alpha$ is choosed by Morozov deviation principle or by GCV $[8,9]$.

In the numerical computation, Newton method is used as follows:

Step1: Set initial value $\alpha_{0}$, then we compute $f_{0}=f\left(\alpha_{0}\right), f_{0}{ }^{\prime}=f^{\prime}\left(\alpha_{0}\right)$, where $f(\alpha)=\left\|T_{\alpha} f_{\delta}-f_{\delta}\right\|^{2}-\delta^{2}$.

Step2: Compute $\alpha_{1}=\alpha_{0}-\frac{f_{0}}{f_{0}{ }^{\prime}}, f_{1}=f\left(\alpha_{1}\right), f^{\prime}{ }_{1}=f^{\prime}\left(\alpha_{1}\right)$.

Step3: If $\alpha_{1}$ satisfied $|\eta|<\varepsilon_{1}$ or $|\eta|<\varepsilon_{2}$, then $\alpha=\alpha_{1}$;

else go to step 4 .

Where $\eta=\left\{\begin{array}{ll}\left|\alpha_{1}-\alpha_{0}\right| ; & \left|\alpha_{1}\right|<C \\ \frac{\left|\alpha_{1}-\alpha_{0}\right|}{\alpha_{1}} ; & \left|\alpha_{1}\right| \geq C\end{array}, C\right.$ is a control constant.

Step4: if $f_{1}{ }^{\prime}=0$, then over,

otherwise alternate $\left(\alpha_{0}, f_{0}, f_{0}{ }^{\prime}\right)$ by $\left(\alpha_{1}, f_{1}, f_{1}{ }^{\prime}\right)$, then go to Step1.

\section{Numerical Result}

In this section, the regularization strategy is applied above to the numerical differentiation, and discussed the implementation of the numerical method and the tests which it performed in order to investigate the accuracy and stability of the numerical differentiation procedure[10,11].

In the examples, the exact data function is denoted by $f(t)$ and the noisy function $f_{\delta}(t)$. $f_{\delta}(t)$ is obtained by adding an random error or an high frequency disturbance error to $f(t)$. 
That is $f_{\delta}\left(t_{i}\right)=f\left(t_{i}\right)\left(1+\delta \sigma_{i}\right)$, where $t_{i}=i h ; i=0,1,2 \cdots \cdots n ; n h=1$, and $\sigma_{i}$ is a uniform random variable with values in [-1,1], such that $\max _{0 \leq i \leq n}\left|f_{\delta}\left(t_{i}\right)-f\left(t_{i}\right)\right| \leq \delta$ or $f_{\delta}\left(t_{i}\right)=f\left(t_{i}\right)\left(1+\delta \sin \left(\omega t_{i}\right)\right)$, where $t_{i}=i h ; i=0,1,2 \cdots \cdots n ; n h=1$, and $\omega$ is a perturbation frequency.

Example 1: The first example is rather oscillatory on $[0,1]$. We choose $f(t)=\sin (10 \pi t)$ and the exact derivative $f^{\prime}(t)=10 \pi \cos (10 \pi t)$. In table1, we give the error between the exact derivative of $f(t)=\sin (10 \pi t)$ and the solution obtained with the regularization strategy. In order to illustrate the numerical approximation to the derivative $f^{\prime}(x)$ denoted $f_{\delta}^{\prime}(x)$, error $_{2, h}=\left(\frac{1}{n} \sum_{i=1}^{n}\left|f_{\delta}^{\prime}\left(t_{i}\right)-f^{\prime}\left(t_{i}\right)\right|^{2}\right)^{\frac{1}{2}}$ is applied.

Table 1 . This is the the error between the exact derivative and regularization derivative

\begin{tabular}{|c|c|c|c|c|}
\hline & error & & & \\
\hline \multirow{2}{*}{$\delta$} & $n=100$ & $n=100$ & $n=200$ & $n=200$ \\
\hline & $\omega=100$ & $\omega=200$ & $\omega=100$ & $\omega=200$ \\
\hline 0.001 & 0.49067 & 0.49201 & 0.11878 & 0.12708 \\
\hline 0.010 & 0.58587 & 0.55242 & 0.45855 & 0.42768 \\
\hline 0.050 & 1.70032 & 1.32859 & 2.24404 & 3.25952 \\
\hline 0.100 & 3.36879 & 2.78627 & 4.52232 & 3.27416 \\
\hline
\end{tabular}

Example 2: The exact function $f(t)=\operatorname{normcd} f(t, 2,3)$, solve $\rho(x)$, such that $\int_{-\infty}^{+\infty} \rho(t) d t=f(t)$.

The complete algorithm for the regularization strategy is as follows:

Step1: Provide the exact discrete data $f_{i}(t)$ of $f(t)$ by MATLAB.

Step 2: Obtain the noisy function $f_{\delta}\left(t_{i}\right)$ by adding a $\delta$ high frequency disturbance error to $f\left(t_{i}\right)$.

Step 3: Compute the approximate solution $\rho_{\delta}(x)$ of $\rho(x)$.

Step 4: Compare $\rho_{\delta}(x)$ with $\rho(x)$.

In experience the exact $\rho(x)$ and error function error are given as follow:

$$
\rho(x)=\frac{1}{2 \sqrt{2 \pi}} \exp \left(-\frac{2}{2 \times 2^{2}}(t-3)^{2}\right), \text { error }=\sqrt{\frac{1}{n} \sum_{i=1}^{n}\left(\rho\left(t_{i}\right)-\rho_{\delta}\left(t_{i}\right)\right)^{2}} \text {. }
$$

Table 2. This is the the error between the exact derivative and regularization derivative( $\omega=217)$

\begin{tabular}{lccl}
\hline & \multicolumn{3}{l}{ error $(\omega=217)$} \\
& $\delta=0.1$ & $\delta=0.01$ & $\delta=0.001$ \\
\hline regularization stratery & 0.1231 & 0.0123 & 0.0012 \\
bisection method & 0.1428 & 0.0143 & 0.0014 \\
interpolation & 2.5203 & 0.2520 & 0.0252 \\
spline & 0.3270 & 0.0327 & 0.0033 \\
extrapolation & 0.2025 & 0.0202 & 0.0020 \\
discrete regularization( $\alpha$ unknown) & 0.0143 & 0.0022 & $7.2694 \mathrm{e}-004$ \\
discrete regularization( $\alpha$ known) & 0.0133 & 0.0021 & $7.2634 \mathrm{e}-004$ \\
\hline
\end{tabular}


Table3. This is the the error between the exact derivative and regularization derivative $(\omega=100)$

\begin{tabular}{lccc}
\hline & \multicolumn{2}{l}{ error $(\omega=100)$} \\
& $\delta=0.1$ & $\delta=0.01$ & $\delta=0.001$ \\
\hline regularization stratery & 0.3215 & 0.0322 & 0.0032 \\
bisection method & 0.2261 & 0.0226 & 0.0023 \\
interpolation & 2.2087 & 0.2209 & 0.0221 \\
spline & 0.5400 & 0.0540 & 0.0054 \\
extrapolation & 0.3434 & 0.0343 & 0.0034 \\
discrete regularization( $\alpha$ unknown $)$ & 0.0126 & 0.0020 & $6.1855 \mathrm{e}-004$ \\
discrete regularization( $\alpha$ known) & 0.0130 & 0.0023 & $6.1835 \mathrm{e}-004$ \\
\hline
\end{tabular}

\section{Conclusions}

In many practical problems, it is sometimes necessary to evaluate the derivative of function whose values are given approximately. Firstly, the problem of estimating the derivative of a function observed with error is studied. It presents a proper regularization strategy and explain how to choose regularization parameter. Secondly, the regularization strategy above to the numerical differentiation is applied and discussed in the implementation of the numerical method and the tests which it has performed in order to investigate the accuracy and stability of the numerical differentiation procedure. Finally, some numerical examples will further illustrate that this method is reasonable, effective and reasonable[12].

\section{Acknowledgements}

The paper is supported by the National Natural Science Foundation of China.(Grant No. 11301132, No. 10701031, No.11171087, No.11001072).

\section{References}

[1] A NTikhonov, On solving incorrectly posed problem and method of regularization, Dokl, Acad, Nauk USSP, 1963, 151(3), 501-504.

[2] Dinh Nho Hao, A mollification method for ill-posed problems, Numer. Math. 1994, 68,469-506.

[3] W Q Liang, Y F Wang and C C Yang, Determining the finite difference weights for the acoustic wave equation by a new dispersion-relationship-preserving method, Geophysical Prospecting, Vol. 63, 11-22, 2015.

[4] J T Zhao, Y F Wang and C X Yu, Diffraction imaging by uniform asymptotic theory and the double exponential fitting, Geophysical Prospecting, Vol. 63, 338-353, 2015.

[5] J J Cao and Y F Wang, Seismic data restoration with a fast L1 norm trust region method, J. Geophys. Eng., Vol. 11, No. 4, 045010, doi:10.1088/1742-2132/11/4/045010, 2014.

[6] W Q Liang, Y F Wang and C C Yang, Comparison of numerical dispersion in acoustic finite-difference algorithms, Exploration Geophysics, http://dx.doi.org/10.1071/EG13072, 2014.

[7] C X Yu, Y F Wang, J T Zhao and Z L Wang, Double parameterized regularization inversion method for migration velocity analysis in transversely isotropic media with a vertical symmetry axis, Geophysical Prospecting, Vol. 62, 1040-1053, DOI: 10.1111/1365-2478.12117, 2014.

[8] Pullia A,Riboldi S. Time-domain simulation of electronic noises. IEEE Transactions on Nuclear Science,2004,51(4),1817-1823 
[9] Vinagre B M, Chen Y Q. Fractional calculus application in automatic control and robotics. 41st IEEE CDC ,Tutorial Workshop 2, Las Vegas,2002

[10] Pocco A,West B J. Fractional calculus and the evolution of fractal phenomena Physica A, 1999,265(3 4),535-546

[11] Ahmad W M,Harb A M. On nonlinear contral design for autonomous chaotic systems of integer and fractional orders. Chaos,Solitions and Fractals, 2013,16,1-9

[12] Magin R L. Fractional calculus in bioengineering,critical reviews in biomedical engineering. Begell House Publications , 2004,32,1-378 